\title{
Methodology for Estimating of Adequacy Concerning Mathematical Models of Complex Objects
}

\author{
Oleg N. Dmitriev \\ Moscow Aviation Institute - National Research University (MAI), \\ Volokolamskoe Highway 4, 125993, Moscow, Russia
}

\begin{abstract}
Conceptual constructions of management quality and adequacy for the mathematical model of an object within management are correlated. Deterministic, stochastic and uncertain situations are localized in the estimation of adequacy. The article exposes the expediency of model adequacy optimization. It identifies the most essential features of the models and objects for the adequacy estimations. We offered a typology of problems for adequacy estimation involved in mathematical models with the formalization, which examines the most problematic issues of adequacy estimating in the considered area.
\end{abstract}

Keywords - adequacy, estimation, management, model, optimization.

\section{Introduction}

One of the most important requirements for optimal managerial decisions related to the management of complex organizational, economic and technical objects is the accuracy requirement arising from the high responsibility of these decisions. Their critical importance exists for decision makers or other persons, including subsequent consequences of adoption and implementation.

DOI: $10.18421 /$ TEM93-25

https://doi.org/10.18421/TEM93-25

Corresponding author: Oleg N. Dmitriev, Moscow Aviation Institute - National Research University (MAl), Moscow, Russia

Email: olegdmitriev@yandex.ru

Received: 01 May 2020.

Revised: 14 July 2020.

Accepted: 20 July 2020.

Published: 28 August 2020.

(cc) BY-NC-ND(C) 2020 Oleg N. Dmitriev; published by UIKTEN. This work is licensed under the Creative Commons Attribution-NonCommercial-NoDerivs 4.0 License.

The article is published with Open Access at www.temjournal.com
As a rule, this accuracy is correlated either with the outputs of the managed object, or with its inputs, but in the end, we should evaluate it outputs.

The accuracy is not associated with managerial decisions, but only with optimal ones, as it is normal not to use this refinement regarding optimality. It is incorrect to discuss the accuracy or inaccuracy of an arbitrary version of a managerial decision. Moreover, it looks unnatural in relation to an insolvent managerial decision.

However, when constructing a mathematical model of a complex managed object, these optimal managerial decisions are obviously unknown. Therefore, depending on the specifics of the managerial situation, any managerial decision in the general case may be optimal.

The optimal managerial decision is recognized as absolutely accurate if, when implemented in the form of a managerial impact, the state indicators and optimization criterion of the managed object will be the best of the admissible and insignificantly different from those assessed when substantiating the optimal managerial decision.

Based on the general constructions of the theory of automatic control, it seems legitimate to assert that the accuracy of managerial decisions when substantiating them, if we exclude errors induced by other tools when making them by the decision maker, it is determined by the accuracy of the operator identification of the managed object in terms of the laws of its state and its external dynamics environment - the adequacy of the corresponding model. As for the construction of the theory of automatic control, the transfer function of the controller with the correct synthesis will always include the transfer function of the managed object [1], [2]. On the resource www.rsl.ru there are also more than 350 references to the relevant literature, which are mostly educational and methodical in its nature.

There is no reason to doubt that this proposition is applicable to management systems of an arbitrary kind, including those that are not described by 
classical transfer functions [3], [4], although it would be worth trying to prove strictly mathematically [5].

With an incorrect synthesis of the managing system, the above statement will be false. For example, the decision maker can completely ignore the realities of the managerial situation and go beyond the field of the analyzed and indicated preferred options for managerial decisions. However, this is already an area of the "incorrect" or "absurd" management, which is not considered in this study.

In the framework of this study, we focus only on the state of the managed object, which is identified by some mathematical model. Of course, the object may not be the managed object, for example, only the object of observation, but in essence the above constructions and calculations of this essentially do not change anything. Naturally, mathematically identified in the form of models are mainly managed objects, either real ones or those that are supposed to give this status.

In turn, this accuracy is associated with the cost of synthesizing a mathematical model. As a rule, increasing the accuracy of identifying an object with a mathematical model in an excellent degree increases the cost of implementing a project for the synthesis of this mathematical model of the managed object. Accordingly, the dependence in the coordinates "accuracy - costs" has gaps of the second kind along the axis "costs" when moving along the axis "accuracy" to the right. However, when moving to the left, costs can reach zero only at the point of absolute inaccuracy, and even that is not necessary. As a rule, the approach to this discontinuity comes already at a distant approach to the threshold of full accuracy, which in principle is unattainable.

Losses from erroneous or insufficiently accurate managerial decisions can be significant and sometimes devastating. The costs of preparing managerial decisions are quite sensitive to their accuracy. Absolutely adequate and inadequate models cannot exist, the problem is only the degree of inadequacy of the mathematical model.

Despite a number of fundamental differences within varieties of formal logical descriptions (models and algorithms, analytical and simulation models), they have a factor-response nature, or, in other words, they establish a mechanism for linking exogenous and endogenous variables, and the nature of output changes when the input is varied.

The increasing complexity of modern management realities requires not only improving the methods for assessing the adequacy of mathematical models of managed objects, but also significantly develop the conceptual foundations of this type of assessment, sometimes requiring an extension of the subject area of adequacy assessment.

\section{Methodology}

The methodological basis of the study was as follows:

- system analysis;

- general control (management) theory;

- theory of mathematical identification;

- probability theory;

- uncertainty theory;

- mathematical statistics;

- optimization theory;

- decision making theory;

- set theory;

- information theory etc.

\section{Results and Discussion}

\section{Background, Applicability and Portability of Known Results}

The problems which include modelling of objects and assessing adequacy are the problems of all period of human existence.

In modern conditions, the problem of evaluating the adequacy of mathematical tools should be given a lot of attention, but in practice quite often both developers and management personnel adhere an inappropriate scheme for obtaining and consuming management tools "as is" in this case.

It is not possible to identify sources with a direct mention of the phrase "adequacy of the mathematical model" in the Russian State Library on the website www.rsl.ru (hereinafter the access date is August 17, 2019). In the Library of Congress of USA on the website www.loc.gov, there are also no direct references in English writing, however, publications with an "internal" context on the subject under consideration are highlighted [6], [7].

A rather significant number of specialized publications is found on the website www.pubsonline.informs.org [8], [9]. However, these publications discuss local and, most often, completely utilitarian technologies for rather narrow areas selected by the authors. An interesting detail is on the INFORMS' website [10]: there is no thematic section "Adequacy".

Analysis of thematic publications (keywords such as "Adequacy of Mathematical Models" and similar) on the resource https://link.springer.com for 20092019 allowed to reveal only one profile of fundamental publication [11], devoted primarily to formalized interpretations of the concept of "adequacy of mathematical models" and partly - its comparison. In the remaining more than 600 supposedly specialized publications, the problems of adequacy of the specific mathematical models and formalizations presented by the authors are mainly in passing touched upon. 
Almost a similar picture we see when analyzing the database on the resource https://scopus.com. However, here the main part of the supposedly specialized publications (there are about 1400) is associated with technical areas and, accordingly, technical objects-mechanics, chemistry, physics, etc. and again, in the relevant publications, the problem of assessing the adequacy of mathematical models is addressed largely as a passing and clearly not a priority. Perhaps the exception is the following publication [12], however, it seems quite abstracted from the specifics and features of the objects.

Meanwhile, in the literature about mathematical statistics [13], [14]. there are many descriptions of methods for testing hypotheses about the statistically significant closeness of statistics or even their belonging to the general population, most often there are estimates of mathematical expectations and, less often, modes from samples of rather ambiguous representativeness. In addition, sometimes the problems of the censored samples [15] and comparison of the statistics obtained on them with regard to deviations from each other are considered.

When evaluating the adequacy of mathematical models, the time series of numerical values are compared in the complex or in pairs first (on the model and on the object for similar time points), and then in the complex at all points limited by the range of observation of the trajectories.

So, the most common and most widely described methods in the literature are:

- statistical pairwise comparison of time series points with the subsequent threshold "vote" according to the proportion of statistically close (for example, with $90 \%$ of the proximity, the presence of adequacy is recognized);

- integral comparison (value of the "area" between the rows is estimated and compared with the threshold value);

- use of various specialized "statistics" and rules such as criteria for statistical heterogeneity and consistency, for example, $\chi^{2}$-Pearson special criterion rule;

- expert comparison of trajectories;

- resolutions of problems with inaccurate estimation of inputs and outputs [16].

- However, their applicability is limited in the area under consideration, because sometime the series have:

- loss points (losses);

- statistical and other heterogeneities, as a result the series including cannot be recognized as ergodic;
- bifurcation branches [17];

- not only stochasticity, but also uncertainty;

- lack of synchronization of the points of temporary reference values.

In addition, mathematical models, as a rule, are not ranked in their own set ("one object - one model" scheme is considered). In the sphere of, for example, economics, they cannot be focused on reproducing experiments with real objects of control. Experiments in providing an assessment of the adequacy of mathematical models are not very often feasible on real objects.

In some sources, software verifiers or computer testers, which make it possible to detect software incorrectness, are indirectly mentioned mainly without specifying. Such systems are widely used in the activities of Microsoft ${ }^{\mathrm{TM}}$ Inc. - mathematical, full-scale and semi-natural. This toolkit is aimed at identifying the logical and characteristic incorrectness of software in applications and operating systems.

Descriptions of optimization problems for mathematical models of managed objects in terms of formulation and solution have not been identified in the available literature.

Accordingly, the following things can be transferred to the considered area:

- methods for comparing "simple time series" (absolutely synchronous, and, as a rule, stochastic, for which ergodicity is characteristic);

- general comparison schemes (comparisons).

Positioning the Relationship of the Mathematical Model of the Managed Object with the Control Functions

As it is well known, the functionality of the managing system is variable within certain limits.

However, at the same time, the mathematical model of the managed object is involved in the implementation of at least one function, the function of optimizing managerial decisions (Figure 1.).

In the Figure 1, one-sided arrows show the sequence of implementation of control functions, and two-sided arrows show the interaction of functional blocks and the mathematical model of the managed object. The dotted lines, which include the last arrows illustrates the possibility of invoking the mathematical model of the managed object, and the solid character is the obligatory nature of this involvement. 


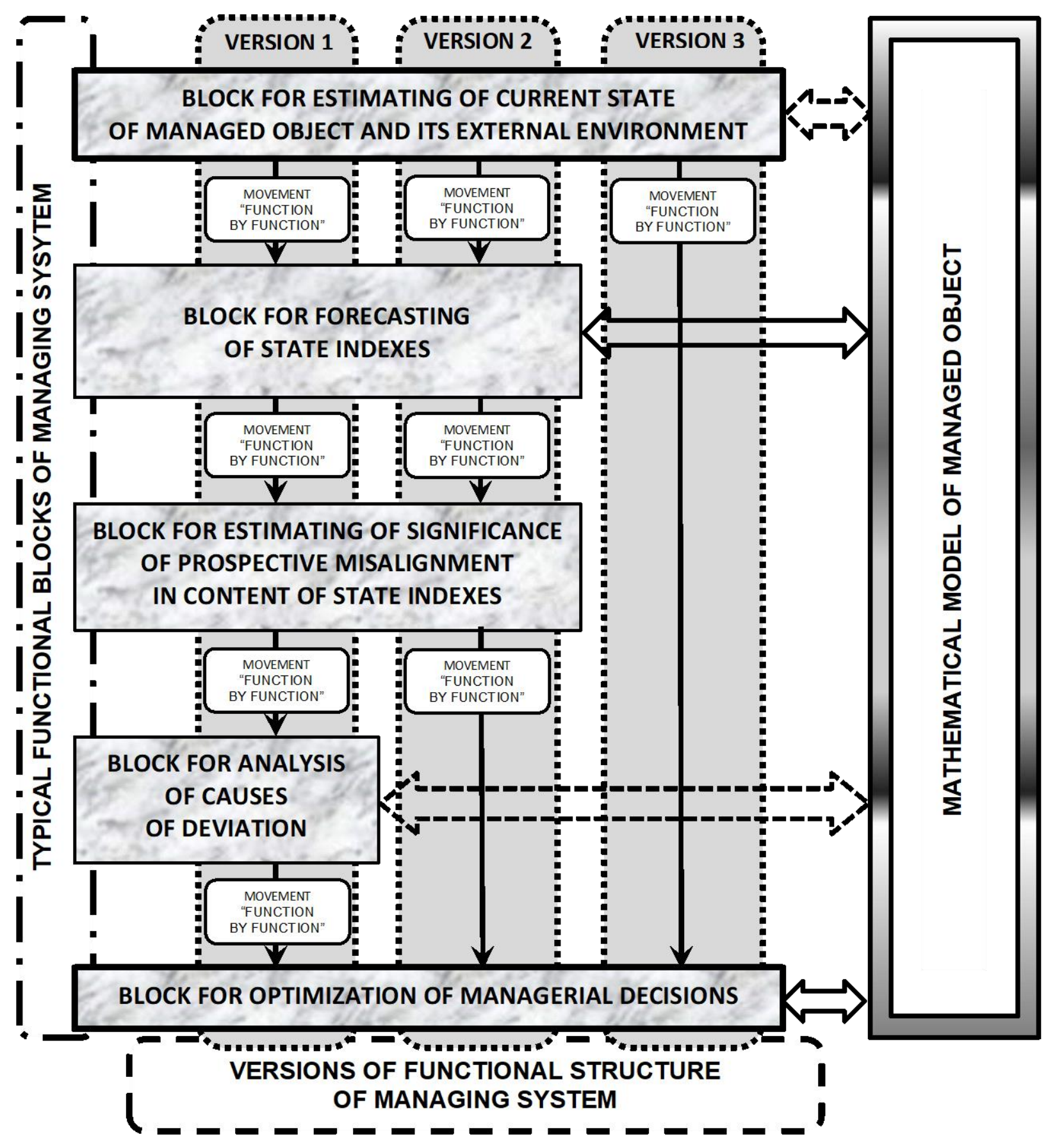

Figure 1. Correlation of the functional blocks of the information-advising managing system with a mathematical model of the managed object

Criticality that Concerns the Adequacy of the Mathematical Model of the Managed Object

The accuracy of managerial decisions and the adequacy of the mathematical model of the managed object are critical.

Sensitivity studies for real management facilities have shown that relative error of managerial decisions at the level of $3 \%$ can already rise catastrophic crises, for example, within financial and economic facilities such as modern Russian high-tech enterprises [18]. There are examples of the study of the criticality including such precision [19], [20].

\section{General Scheme for Evaluating the Adequacy} of Mathematical Models

The most important requirement for mathematical models of a managed object is their "proximity" to a real object in the following aspects:

- in terms of the correctness of tracking the inputoutput relationship, the coincidence of the output characteristics of the object and model with identical inputs (physical or informational). Proximity in the indicated sense is accepted classically proper, and it is called adequacy; 
- in terms of the correctness with reference to decomposition of the model description in terms of management objectives. This aspect defines the scheme for breaking the description of an object into local models of the "black box" type, which are then no longer decomposed, remaining elements. All elements of the hierarchical tree of models should be adequate to the corresponding prototype objects.

The coincidence in the above two senses sometimes is called the authenticity of the mathematical model of the managed object. So, in particular, some mechanistic constructions are applied in the formation of the well-known classical physics equations of dynamics in the electromagnetic field by James Clerk Maxwell. There are also known mechanical descriptions introduced in constructions in the field of particle physics, etc. The author also knows other examples of using a similar approach in the study of macro physical processes in outer space. However, even in a school course of physics, the structure of the atomic nucleus appears in the constructions of mechanical systems, rather than interacting fields, i.e. constructions of Isaac Newton are unnaturally incorporated into quantum mechanics.
If the problems of the second aspect are solved mainly now on a heuristic level, then adequacy in the classical sense allows a completely rigorous assessment.

The questions of adequacy assessment are traditionally considered and are relevant, complex and rather far from being resolved.

There are two ways to assess the adequacy of mathematical models and, perhaps, algorithms.

The first one is a one-time procedure, which is an assessment of adequacy as a comparison between the closeness of time series of state indexes of the managed object observed on a real object and obtained during a computational experiment with a software implementation of a mathematical model. A one-time procedure is traditionally implemented for several variants of the source data.

The second is the permanent procedure concerning "reassessment" of adequacy.

In the Figure 2 there is the structural diagram for evaluating the adequacy of the mathematical model (and not just it) of the managed object, where the dashed line indicates the fragment whose adequacy is subject to verification.

The correctness of the conclusion about the adequacy or inadequacy depends on how much the errors in the assessment of state indexes, initial data and the estimation of the significance of the mismatches will be neutralized or compensated.

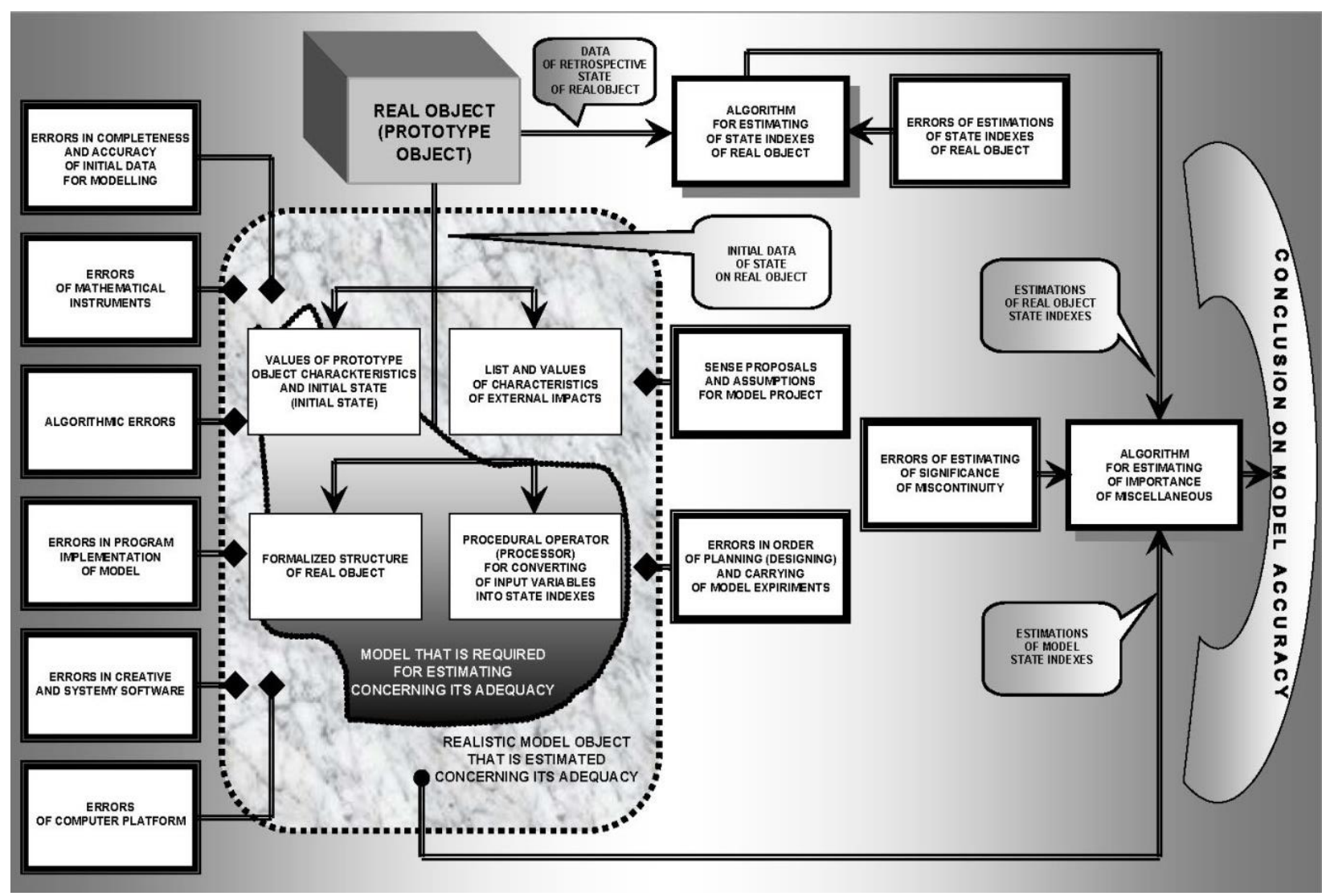

Figure 2. Conceptual framework for estimating (evaluating) the adequacy of software (software implementation of the mathematical model of the managed object) 
The first three types of error characteristics affect the conclusions about adequacy to the greatest degree, while the problem is complicated by the fact that the characteristics of the errors listed are uncertain. Evaluating the adequacy is an attempt to verify the importance of other types of errors and simplifications.

The variation of the proposed procedure is possible, which is used less frequently than indicated, but is also quite common in scientific research. This is an important class of verification experiments in which the real object is replaced by a certified, reference model. As a rule, the "assay" model is much more detailed than being checked for adequacy, and is applicable to a wider set of valid options.

The advantages of the conceptual method under consideration using the "assay" model are quite obvious: reduction of costs, including time, on the assessment of adequacy due to refusal to examine a real managed object, more representative tests for an incomparably wider range of situations. The disadvantages are also seen quite clearly: introduction of additional errors inherent in the "ideal" model, need to fully master the theoretical basis of such a model and practical skills in working with its software implementation.

The second estimate is an integration superstructure over the first.

\section{Typology of the Tasks Involved in Evaluating the Adequacy of Mathematical Models of the Managed Object}

The adequacy analysis procedures depend on the nature of the model or algorithm, that is the outputs of the models are compared with the responses of the real or replaced by the specified method of the object, and in this sense, they correspond with the type of environment.

The following cases are possible when evaluating the adequacy of the mathematical model of the managed object: the case of a deterministic medium, the case of a stochastic medium, and the case of an uncertain environment. In the case of the presence of typological combinations (heterogeneity among the components with regard to the nature of the medium), its integral type is determined by the most "complex" medium. That is, in the presence of at least one stochastic component, along with the determinate medium, the medium is recognized as stochastic, and in the presence of at least one uncertainty, it is generally uncertain.

When evaluating the adequacy of mathematical models of the managed object, the following three categories of tasks can be considered:
- assessment of the absolute level of adequacy;

- assessing the acceptability of adequacy;

- comparative assessment of the level of adequacy, ranking of mathematical models by the level of their adequacy. This task may have the nature of unscaled ranking (according to the "places" of adequacy, the first, second, third, etc. places have an ordinal space), or maybe of a scaled ranking, when the mutual removal of ranked models on the adequacy scale is known, there is a metric space.

However, scaling of the latter type bring serious problems, if the adequacy estimates are heterogeneous (deterministic, stochastic and uncertainty), then their comparison raises unresolved methodological problems itself, because universal metric spaces are still completely unexplored by science.

The considered task of evaluating the adequacy also varies depending on what state indexes of the managed object are being evaluated.

So, there are the following options: assessment by a scalar and a vector state index of the managed object.

Accordingly, problems arise here are similar to the problems of multicriteria optimization. The mathematical model may be adequate for all state indexes or one part of it. In the same way, it may be adequate at some time periods or maybe not, or it may be adequate under certain conditions and inadequate under others.

\section{Task Statement of the Adequacy Assessing of the Mathematical Model of the Managed Object}

There is a problem of evaluating the adequacy of a model as an arbitrary type to a real prototype object.

The problem reduces to testing the two-alternative hypothesis $\boldsymbol{H}_{0} / \boldsymbol{H}_{\boldsymbol{l}}$ concerning the outputs of the mathematical model and the real object coincide in the sense corresponding to the medium ( deterministically, stochastically or uncertainly), that is, the resulting difference between them is insignificant for the same input action, for which the identification of the mathematical model was carried out.

We can consider a method of testing the hypothesis that the outputs of the model and the real object coincide in a sense, that is, the resulting difference between them is insignificant with the same input action for which the model was identified. As it is shown in the Figure 3, if we approach the problem in an abstract way, it would be worth comparing the outputs of the $\boldsymbol{Y}^{M}$ model (hereinafter they are vectors) and the outputs of the $\boldsymbol{Y}^{\boldsymbol{O}}$ object (that is, the closure of points $\boldsymbol{M}_{2}$ and $\boldsymbol{O}_{2}$ to 
the comparator is implemented), but one can compare observed on the model output $\boldsymbol{Y}^{\boldsymbol{M} \boldsymbol{M}}$ and observed on the real object output $\boldsymbol{Y}^{M O}$, whereby the input of the real object is given the real impact of $\boldsymbol{X}^{\boldsymbol{M} O}$, and the input of the model is simulated by the influence of $\boldsymbol{X}^{\boldsymbol{P}}$, and identification on the model is done by the set $\left\{\boldsymbol{X}^{M O}, \boldsymbol{Y}^{M O}\right\}$. This circumstance leads to some additional error, which is unobservable and non-exclusive.

We assume that the studies are carried out on a certain period $t \in\left(\boldsymbol{T}_{\text {beg. }}, \boldsymbol{T}_{\text {end }}\right]$, for which all discrete points of the time series of state indexes within the mathematical model and the managed object are located inside this period.

Since the management process is deliberately discrete, only the following samples of output processes can be compared for this time interval:

$$
\left\{\begin{array}{l}
Y_{\text {sampl. }}^{M M} \equiv\left\{Y^{M M}\left(t_{11}\right), \ldots, Y^{M M}\left(t_{1 n}\right)\right\} ; \\
Y_{\text {sampl. }}^{M O} \equiv\left\{Y^{M O}\left(t_{21}\right), \ldots, Y^{M O}\left(t_{2 m}\right)\right\} .
\end{array}\right.
$$

In the general case, of course, $\boldsymbol{n} \neq \boldsymbol{m}$, and the sequences being compared can be tied to different instants of time; moreover, in the general case, the set of instants of time $\left\{\boldsymbol{t}_{\boldsymbol{1}}\right\}$ has an empty or insignificant intersection with the set of instants of time $\left\{\boldsymbol{t}_{2 j}\right\}$. There are the situations when in the series $\boldsymbol{Y}_{\text {sampl. }}^{\boldsymbol{M O}}$ there is some thinning.
The hypothesis should be verified: $\boldsymbol{H}_{0}: \boldsymbol{Y}_{\text {sampl. }}^{M M_{\text {sampl }}} \stackrel{\Delta}{\neq} \boldsymbol{Y}_{\text {sampling }}$ alternative hypothesis: $\boldsymbol{H}_{l}: \boldsymbol{Y}_{\text {sampl. }}^{M M_{\text {sampl. }}} \stackrel{\Delta}{=} \boldsymbol{Y}_{\text {sa }}^{\text {or }}$ vice versa, where symbol $\stackrel{\Delta}{=}$ is the equality by definition, for example, in a statistical sense; but symbol $\stackrel{\Delta}{\neq}$ is the inequality by definition, for example, in a statistical sense.

The first step in evaluating the adequacy is always the synchronization of time scales according to $\boldsymbol{Y}_{\text {sampl. }}^{M M}$ and $\boldsymbol{Y}_{\text {sampl. }}^{M O}$

When checking the statistical hypotheses $\boldsymbol{H}_{\boldsymbol{0}} / \boldsymbol{H}_{\boldsymbol{l}}$, if there is a statistical situation, as known, errors of the first and second kind can be made.

In relation to the problem under consideration, they are interpreted as follows:

- error of the first kind: the conclusion is drawn about the inadequacy of the model, while in reality it is adequate, the probability of this event determines the significance level $\alpha$,

- error of the second kind: the model is actually inadequate, the opposite position is accepted, the probability of this event is $\beta$, which determines the power of the criterion, which corresponds to the level 1 1- $\beta$.

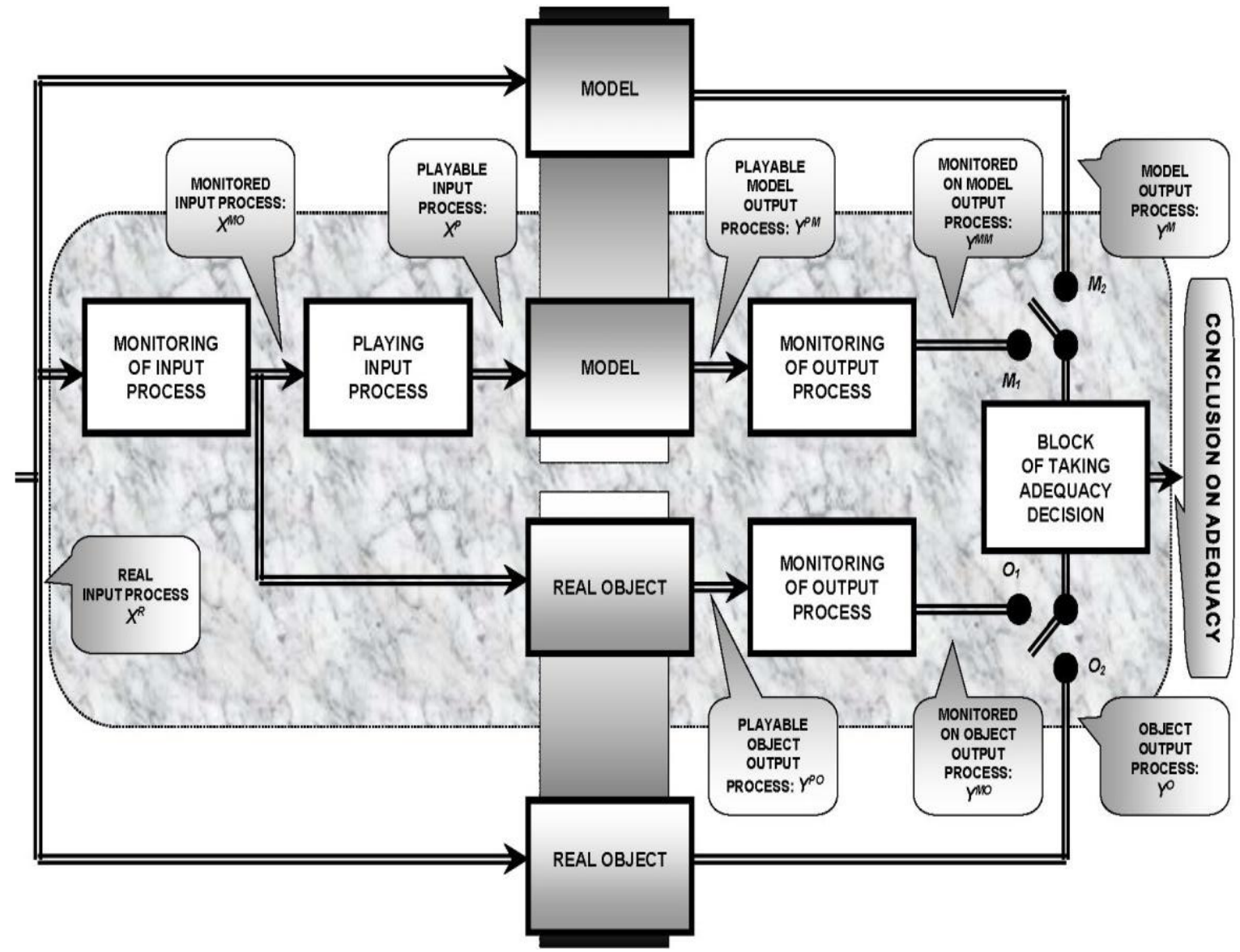

Figure 3. Specifics of the semantic content of the adequacy estimating 
It is well known that with a fixed sample size, a simultaneous decrease in values $\alpha$ and $\beta$ is impossible to achieve, therefore it is advisable to choose a procedure that minimizes the value $\beta$, since the losses from a second-type error, as it was already indicated, are much larger than from first-type errors. The use of an incorrect, inadequate model entails significantly higher costs than additional analysis and refinement of the model, even if the model already constructed actually gives a high-quality, and acceptable adequate description. In this case, it is advisable to solve the problem of minimizing the average risk in the traditional interpretation. A similar task was considered.

There are other known adequacy assessment procedures. One of them involves evaluating balances, for example, cash flow balances for some discrete time points. Such estimation is used, for example, for financial and economic potential models that include institutional localizations.

Obviously, for the case of a deterministic environment, the comparison between the outputs of the object and its model is based on some deterministic measure.

For the case of uncertain mathematical models, methods for assessing their adequacy have failed to be identified among the developments available for study or to develop original ones. The only thing that now does not cause catastrophes in this case is the small-scale experience of the synthesis of uncertain mathematical models, they are already used [21], [22], but not too widely. Of course, the well-known approach based on the reduction of uncertainties to stochasticity remains fundamentally applicable, but the correctness of its application has not been seriously studied.

Comparison of time series of two types, uncertainty and stochastic, also seems to be a completely unexplored area. So, very often the data of the state indexes of the managed object are uncertain, but estimates by the mathematical model are deterministic or stochastic.

We can consider how to present the problem referring to optimizing the level of adequacy of the mathematical model of an object now (as a rule, managed object).

Some established indexes of the managed object are introduced, that is, a vector state index $\vec{I}\left(\boldsymbol{t}_{i}\right) ; \boldsymbol{i} \in$ $[1, T]$.

Accordingly, there arises a non-trivial, practically not considered, in the areas of fundamental and applied operations, task of complex optimization of design decisions to ensure an acceptable level of adequacy concerning the used model of the managed object \{Adequacy of model\} and profile managerial decisions in relation to the managed object \{Project activities\}:

\section{$\vec{I}\left(t_{i}\right) \rightarrow$ extr $\{$ fixed type: $\underline{\min }$ or $\underline{\max }\} \quad ; i \in[1, T]$, \{Adequacy of model\} \& \{Project activities\},}

where $\boldsymbol{T}$ is the number of discrete time points on the selected management period.

By the way, the conclusion about the adequacy of the model depends on the length of the period for which it is being evaluated.

This problem is solved after its correct scalarization by one of the known or specially synthesized methods.

\section{Implications After Evaluating the Adequacy of the Mathematical Model of the Managed Object}

In general, the following three alternatives can be considered:

- use of an adequate mathematical model;

- refusal to use a mathematical model if it is recognized as inadequate;

- use of an inadequate mathematical model.

The non-use of an adequate model looks irrational, although such cases are possible if, in particular, personnel support the functioning of the mathematical model of the managed object is difficult, for example, a specialist is ill. In practice, the author also encountered exotic situations when, with an adequate mathematical model, its developers, instead of using it, urgently "drew" supposedly calculated numbers "from the head", hoping that nobody would be able to verify them.

In some cases, the use of an inadequate mathematical model is a productive scientific and design solution, it allows us to narrow the scope of management and accumulate managerial experience.

In general, the task of optimizing the adequacy should be posed and solved considering the costs of ensuring adequacy.

There are three classes of problems referring to assessing the adequacy of the mathematical model of the managed object:

- classical task of matching for "object - model";

- task of comparing the comparative adequacy of models;

- task of optimizing the adequacy of the model.

Only the first and the second alternatives are considered, but this is wrong, since in some cases the use of a "bad" model is preferable to not using any one, for example, when forming an argument. 


\section{Procedural Execution of the Classical Adequacy Assessment}

We formulate the requirements that the adequacy assessment procedure has to meet in the context of the hypothesis testing scheme $\boldsymbol{H}_{0} / \boldsymbol{H}_{\boldsymbol{1}}$ :

- it is necessary to consider the stochastic nature of the observed output processes of the object and its model;

- deterministic component of the output process in the general case can change over time, that is, the corresponding processes are non-stationary;

- in order to achieve analysis flexibility, a conclusion like "model is adequate" or "model is inadequate" should be preceded by a concrete numerical measure of the correct and erroneous solutions (for example, the value of $\alpha$ ) and only then compare the values of $\alpha$ with a given level of $\alpha_{f i x}$, accepting or rejecting the hypothesis for a fixed significance level $\boldsymbol{\alpha}_{f i x}$. This premise, with all its correctness, leads to a certain complication of the algorithms, since in addition there arises the problem of numerical estimation of distribution functions;

- based on considerations of the economics of research, one should focus on the most compact samples of the output data regarding the model and real process, and the number of model samples should also be reduced if possible, focusing on ergotized criteria. Considerations for minimizing the values of $\boldsymbol{\alpha}$ and $\boldsymbol{\beta}$ require the opposite, that is, a compromise should be sought, for example, following the path of iterative "fitting" (additional inclusion in the set) including new sample elements;

- if possible, abstract from the type of confidence function.

The most appropriate is the choice in favor of a set of procedures that allow the entire spectrum of possible situations to solve the problem, as well as verify the adequacy on the basis of several procedures. In other words, the concept of a polyalgorithmic assessment of the adequacy of models is also productive (and perhaps the only one applicable).

The most correct way to assess the adequacy, which was found to be appropriate, is to use the following well-known methods:

- set of methods for assessing statistical inconsistencies;

- $\chi^{2}$-Pearson criterion rule;

- Kolmogorov-Smirnov criterion rule;

- criterion rule based on Bonferroni $\boldsymbol{t}$-statistics, as well as some others.
Comparison tools built into standard statistical packages can be used, for example, systematized in the Matlab ${ }^{\circledR}$ software medium.

Computational experiments to assess the adequacy of mathematical models and algorithms are to be planned. This planning is aimed at providing the most accurate or acceptable assessment of the level of adequacy, provided that the costs of researching this adequacy are minimized, or the level of such costs is not exceeded for a predetermined value.

The planning of experiments of the analyzed type implies:

- identifying a list of state indexes for which a comparison is planned;

- determination of a specific variant of the object for which the study will be carried out;

- presence of a time interval for which the comparison will be performed, as well as a step of discreteness. It is possible that many specific points can be fixed;

- determination of source data sources and the order of their primary processing;

- establishing a list of special situations whose presence or absence should be checked;

- adoption of validation algorithms;

- regulation of the technical parameters of computational experiments (for example, the number of repeated implementations of a simulation experiment, etc.).

Thus, an experiment to assess the adequacy of a mathematical model or algorithm can be interpreted as planning an experiment with a vector response and a mixed nature of factors (some of them are quantitative, and some are qualitative). Therefore, it is legitimate to use traditional methods of experiment planning, supplemented by informal adjustment.

In conclusion to the discussion on the problem of adequacy assessment, we note that in relation to models and algorithms the principle of presumption of adequacy applies, that is, a model or algorithm is assigned to the category of adequate until proven otherwise. At the same time, the proof of inadequacy creates a stereotype of inadequacy: in the case of at least a single identification of the inadequacy of the model, it can be considered adequate, even if there are no new evidence-based refutations of the hypothesis of model adequacy.

Assessment of adequacy and / or authenticity is often referred to as verification. Sometimes the concepts of adequacy and authenticity are distinguished. In this case, authenticity involves assessing the similarity through the prism of structural and procedural correspondence, which comprises the mechanism of functioning and development of the modelling object to the real object, including structural isomorphism. Adequacy, 
on the other hand, implies the formal closeness of state indexes under external influences that are identical in a sense, and the object of modelling is considered a "black box". With this distinction, a formal paradox is possible: an inauthentic model may be adequate under certain conditions, which is often observed both in practice and in theory, when the use of the wrong method leads to the correct result. However, in a more correct presentation, verification also involves checking the operability of the software product. In this sense, the appraisal of the adequacy of a "non-launching" software product is a typical example of an incorrect evaluation or optimization management task of a design type.

The conclusion about the adequacy or inadequacy is parameterized by the degree of rigor of assessing the presence or absence of this model property.

Details of the algorithmic execution of the adequacy procedure for the case of stochastic objects and simulation models are given.

\section{Development Approbation Experience}

The development described in detail by the author was successfully applied for assessment [23]:

- adequacy of a multi-purpose simulation model of the state of the fleet within final and completing products of mainly aviation equipment;

- comparative adequacy of a number of simulation and analytical models of a similar nature;

- permissibility of scaling experiments with the specified simulation model (proportional reduction in the number of the final product park);

- assessing the adequacy of the analytical model of the state of the financial and economic potential within high-tech enterprises and corporate structures.

\section{Conclusion}

The stated considerations and the results of the study give reason to formulate the following statements, conclusions and recommendations:

1. Problem of assessing the adequacy of the developed mathematical models among complex management objects, including objects of an economic nature, is relevant due to the fact that the quality of managerial decisions is a critical factor in successful management in all areas;

2. Adequacy of a mathematical model is evaluated so that it is evaluated inextricably, and linked with some other information objects, including its software implementation, in the course of preliminary computer experiments on software implementation;

3. Quite a lot of research is known in this area, however, they were mainly focused on rather simplified situations, with stochastic series of state indexes. Meanwhile, at a minimum, estimation methods for uncertain mathematical models are required;

4. Apparatus for evaluating the adequacy of mathematical models of the managed object should be developed for classical comparison cases including the model with the object, for the case of matching the adequacy of models and for the case of optimizing the level of adequacy of the model;

5. Comparable state indexes of the managed object can be deterministic, stochastic and uncertainty. The corresponding time series may not be synchronized. This generalization of the situation gives rise to the need for quite revolutionary projects in order to develop methods for assessing the adequacy of mathematical models of complex managed objects.

6. Adequacy of the mathematical model of the managed object is the subject to polycriteria optimization, not maximization;

7. In the general case, it is advisable in the complex to optimize the adequacy of the model of the managed object and managerial decisions in relation to it. In particular, for each kind of management tasks, a model of the managed object corresponding to its level of adequacy should be applied;

8. Developed and presented technology for assessing the adequacy of a number of complex analytical and simulation models has demonstrated its applicability. 


\section{References}

[1]. J. C. Hsu and A. U. Meyer, Modern Control Principles and Applications (McGraw-Hill, 1968)..

[2]. Yurevich, E. I. (2005). Fundamentals of robotics. St. Petersburg.: BHV-Petersburg.

[3]. Forrester, J. Fundamentals of enterprise cybernetics (Industrial dynamics). Transl. from Engl. Moscow: Progress; 1971. 340 p.

[4]. Wiener, N. (2019). Cybernetics or Control and Communication in the Animal and the Machine. MIT press.

[5]. Raol, J. R., \& Ayyagari, R. (2019). Control Systems: Classical, Modern, and AI-Based Approaches. CRC Press.

[6]. Khuri, A. I. (2009). Linear model methodology. CRC Press.

[7]. Boland, L. A. (2014). Model Building in Economics: its purposes and limitations. Cambridge University Press.

[8]. Gianfreda, A., \& Bunn, D. (2018). A stochastic latent moment model for electricity price formation. Operations Research, 66(5), 1189-1203.

[9]. Greenberger, M. (1980). A way of thinking about model analysis. Interfaces, 10(2), 91-96. https://doi.org/10.1287/inte.10.2.91.

[10]. Pubsonline (2019). Subject classification scheme for the $O R / M S$ index. Retrieved from: https://pubsonline.informs.org/pbassets/ORMSSubjectClassifications1519925344200.pdf. [accessed: 15 March 2020].

[11]. Hey, J. D., Lotito, G., \& Maffioletti, A. (2010). The descriptive and predictive adequacy of theories of decision making under uncertainty/ambiguity. Journal of risk and uncertainty, 41(2), 81-111.

[12]. Coquand, T., \& Huber, S. (2019). An Adequacy Theorem for Dependent Type Theory. Theory of Computing Systems, 63(4), 647-665.

[13]. Pugachev, V. S. (2014). Probability theory and mathematical statistics for engineers. Elsevier.

[14]. Ross, S. M. (2014). Introduction to probability and statistics for engineers and scientists. Academic Press.
[15]. State Standard (1984). GOST of USSR 27.504-84 "Methods of reliability assessment for sensurround samples". Moscow: State standard of USSR Publishing House.

[16]. Song, E., \& Nelson, B. L. (2019). Input-output uncertainty comparisons for discrete optimization via simulation. Operations Research, 67(2), 562-576.

[17]. Baird, B. (1989). A bifurcation theory approach to the programming of periodic attractors in network models of olfactory cortex. In Advances in neural information processing systems (pp. 459-467).

[18]. Zolotova, V. A. (2017). Upravlencheskie problemy i zadachi formirovaniya programmy antikrizisnogo upravlencheskogo innovirovaniya v vysokotekhnologichnoe predpriyatie promyshlennosti Rossii [Management problems and tasks of forming the program of anti-crisis management innovation in the high-tech enterprise industry of Russia] M.: KnoRus. KnoRus, 210, 13.

[19]. Demchenko, O. F. (2011). Metodologiya matematicheskogo modelirovaniya organizatsionnykh struktur aviatsionno-promyshlennogo kompleksa Rossiiskoi Federatsii [Methodology of mathematical modeling of organizational structures for aviation industrial complex of the Russian Federation]. Moscow, KnoRus.

[20]. Dmitriev, O. N. (2017). Strategic problems and directions of developing rehabilitation of managing systems of Russian high-tech complexes. Microeconomics, 6(5), 24.

[21]. Hollis, C, Price, S., Dijkm, H, Wei, L., Frese, D., van Rijen, M. and Al Salhi, M.(2011). Uncertainty in a Giant Fractures Carbonate Field, Oman, Using Experimental Design. In: Ma, Y.Z. \& La Pointe, P.R. (Eds.) Uncertainty analysis and reservoir modelling. American Association of Petroleum Geologists Memoir, 96, 137-157.

[22]. Johansson, R., \& Rantzer, A. (Eds.). (2012). Distributed decision making and control. Springer.

[23]. Dmitriev, O. N., \& Novikov, S. V. (2018). Economic assessment of federal scientific programs. Russian Engineering Research, 38(4), 326-329. 